\title{
Simulation Database System of the Active Ingredients in Compound Decoction of Chinese Medicine
}

\author{
Long Zhang ${ }^{1}$, Hua Zhao ${ }^{2}$, Gang Zhou ${ }^{3}$, Tianshui Niü ${ }^{3}$, Jianshe Yang ${ }^{1,3 *}$ \\ ${ }^{1}$ Lanzhou Institute of Chemical Physics, Chinese Academy of Sciences, Lanzhou 730000, P.R. China \\ ${ }^{2}$ Agricultural College of Gansu Agricultural Unversity, Lanzhou 730070, P.R. China \\ ${ }^{3}$ Life Science College of Northwest Normal University, Lanzhou 730070, P.R. China
}

\begin{abstract}
Compound decoction is the main form of clinical application of Chinese medicine. Current research on active ingredients of Chinese medicine has commonly relied on chemical separation and extraction technology by using organic solvents, which was very different from the traditional method of water-phase boiling. Difference in preparation methods would lead to awareness discrepancy in active ingredients. The present system simulated the decoction boiling conditions of traditional Chinese medicine, and established databases of related components and their interaction, and analysed the possible active ingredients existed in compound decoction via database mining, statistical analysis and other technologies.
\end{abstract}

Keywords: Simulation; Active ingredient; Decoction; Chinese medicine

\section{Introduction}

Compound decoction, the main clinical form of Chinese medicine [1], plays an important role in reflecting the efficacy of Traditional Chinese Medicine (TCM). It is essential and helpful, therefore, to clarify the scientific nature of TCM compound drugs based on exploring the active ingredients of compound decoction, understanding the material basis and the therapeutic principle. Chinese herbal compound is a complex molecular system. During the processing, accompanied by complicated physical and chemical changes, there may be interactions among the compound components, between the component and the solvent, and compounds resulting from component interaction. Thus, the active ingredient analysis of the TCM compound is a very difficult project. If we use the given components information about Chinese medicine, combined with computer simulation techniques to simulate the various physical conditions and chemical reactions production process of TCM compound decoction, which is derived compound decoction of Chinese medicine that may exist in the active ingredients, then the experiment may lead to a significant reduction in manpower and material inputs.

Analysis of effective components of Chinese medicinal methods is currently used primarily for chemical separation and extraction analysis in the liquid, vapour, or supercritical conditions, the use of water, alcohol or other organic solvents. The main component of natural Chinese herbal medicines is then extracted and then purified, and then analysis confirms one of the chemical compositions of traditional Chinese medicine monomer. A lot of research has been conducted, whether a single flavour of natural herbs, or Chinese herbal compound [2-5]. However, the clinical application of traditional Chinese medicine has been studied less than the clinical application of traditional Chinese medicine as compound drugs, a separate single herb extracting the active ingredients of traditional Chinese medicine; Chinese herbal compounds are bound to ignore the interaction between each component role, as well as the complexes formed between components of the interaction. Second, the most widely used traditional Chinese medicine in terms of clinical curative effect of the most precise formulation is the soups, and traditional Chinese medicine decoction of the production process does not use organic solvents. So, the non-water-soluble components of Chinese medicine may not be the real active ingredients, but have become interfering factors. Japanese scholars found that only $3.5 \%$ volatile oil existed in decoction when Banxia-Houpu-compound was prepared with clay pot. They speculated that it may not be volatile oil playing the role of the active ingredient efficacy. Professor Wei provided similar results [6].

Traditional Chinese medicine databases have uses computer technology and bio-informatics technology, and modernization of Chinese medicine is the main research direction [7-8]. Much research has been conducted in this area and there are many professional Chinese medicines. The databases contain very comprehensive information on natural medicines, but these databases are mostly literature based, featuring only information retrieval of Chinese medicine, which cannot be based on the analysis of large amounts of information stored; the function of the databases is relatively simple.

Effective components of a Chinese medicine compound decoction simulation database system includes the existing literature on the basis of scientific research and, uses database mining techniques to simulate the traditional Chinese medicine decoction boiling conditions, as well as tests compound decoction of Chinese medicine derived from active ingredients.

\section{Database system approach}

Active ingredient of Chinese medicine compound decoction simulation system uses the B/S (Browser/Server, Browser/Server mode) structure model, back-end database using SQL Server 2000, and future language using ASP (Active Server Page). B/S structure is popular as

*Corresponding author: Professor Jianshe Yang Ph.D, Lanzhou Institute of Chemical Physics, Chinese Academy of Sciences, Lanzhou 730000, P.R. China Tel: +86-931-4968121; Fax: +86-931-8277088; E-mail: yangjs@impcas.ac.cn

Received September 09, 2010; Accepted November 22, 2010; Published December 29, 2010

Citation: Zhang L, Zhao H, Zhou G, Niu T, Yang J (2010) Simulation Database System of the Active Ingredients in Compound Decoction of Chinese Medicine. J Bioequiv Availab 2: 131-134. doi:10.4172/jbb.1000045

Copyright: $\odot 2010$ Zhang L, et al. This is an open-access article distributed under the terms of the Creative Commons Attribution License, which permits unrestricted use, distribution, and reproduction in any medium, provided the original author and source are credited. 
a web access mode, and its biggest advantage is that the client need not install any special software, just a browser. Moreover, this model simplifies matters for the client, requires zero client maintenance, and users can access the network for as long as they wish. In addition, $\mathrm{B} / \mathrm{S}$ model extensions are very easy to realize, in that the core of the system functions are concentrated on the server to simplify system development, maintenance and use. An active ingredient of Chinese medicine compound decoction simulation system running on the server side means that the user can access the network at any place where they have access to the system, the system implementation inquiries, and analysis.

Chinese herbal compound decoction of the active ingredient simulation system of the establishment of the database back-end database using SQL Server2000 database management system for storage and management does not need a client/server type (client / server, $\mathrm{C} / \mathrm{S}$ ) structure database system, but through the establishment of B/S (Browser/Server, Browser/Server mode) structural model database. The $\mathrm{B} / \mathrm{S}$ structure comes from the rise of a web network structure model, with the web browser being the most important application for the client. This model unifies the client with the core of the system function realization, on the part of the server's to simplifying system development, maintenance and use. One of the biggest advantages of the $\mathrm{B} / \mathrm{S}$ is that one can operate from anywhere without having to install any special software, as long as there is a computer with Internet access can use the client to zero maintenance; expansion of the system is very easy. $\mathrm{B} / \mathrm{S}$ structure is being used more and more, especially the demand-driven AJAX technology. Its procedures can also be part of the client computer processing, thereby greatly reducing the burden on the server and increasing interactivity. Real-time updates can be carried out locally.

Simulation of the active ingredients of Chinese medicine compound decoction of the database system consists of three subdatabase components: first, storage of Chinese medicine chemical composition, solubility, function and content of chemical constituents of Chinese herbal pieces database; secondly, reactions, to store chemical composition of traditional Chinese medicine formula for the chemical reaction library; and thirdly, ingredient efficacy of Chinese medicine compound libraries. The system allows users to enter the drug name and reaction conditions related to the various sub-database searches by drug name index in the chemical composition to the relevant database. This allows the system to retrieve the composition, in combination with anti-temperature, pressure, time and other reaction conditions, chemical reaction search database, corresponding to the reaction products. System architecture diagram was described in (Figure 1).

Simulation of the active ingredients of Chinese medicine compound decoction database system is based on the following data sources: "Chinese Pharmacopoeia" (2005 edition), and China total resource base of knowledge (CNKI), which contains the research results and literature information. The recorded data must be officially published pharmacopoeia in the herbal literature.

\section{Database system interface design}

With regard to effective components of Chinese medicine compound decoction simulation database system to the traditional Chinese medicine decoction boiling method for the simulation object, the main control conditions are: temperature, pressure, boiling number, and boiling solvent, such as traditional Chinese medicine Radix decoction

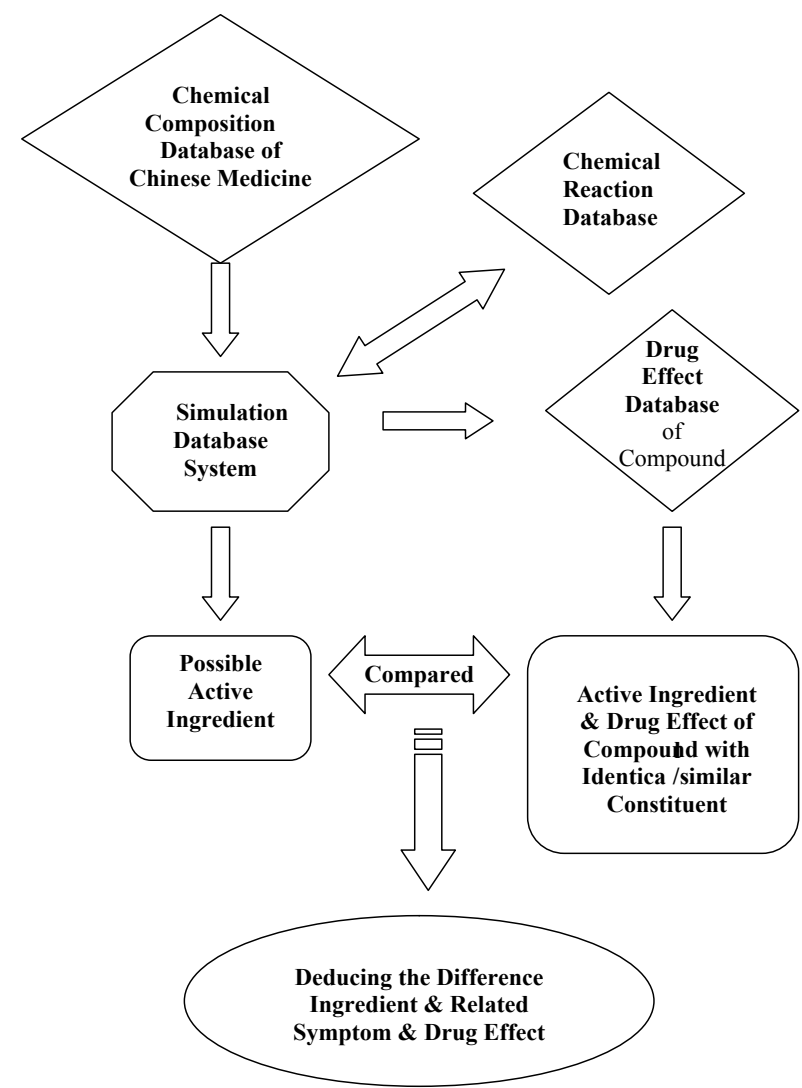

Figure 1: System diagram of simulation database.

of Chinese medicine In the aqueous phase, under the conditions of the environment, it does not use organic solvents, the temperature generally does not exceed $100^{\circ} \mathrm{C}$, pressure in an atmospheric pressure, generally boiling twice, and twice a decoction final mix. Active components of Chinese medicine compound decoction main interface design, with the simulation database system being shown in the (Figure 2).

The main interface includes: components of a single Chinese medicine smell, the number of dosage forms, drug name, dosage, temperature and time control, used to enter the relevant conditions for decoction.

\section{Database applications}

The system uses Chinese herbal compound decoction of the active ingredient simulation database system, classical Chinese medicine prescriptions Sijunzitang analysis, Sijunzitang from ginseng (9 grams) Atractylodes (9 grams) Poria (9 grams) Baked Licorice (6 grams), with main functions as follows: [9-10] adjust the gastrointestinal function; enhance immune function; the promotion of bone marrow hematopoietic function, accelerating the formation of red blood cells. The results are in (Figure 3).

Through a systematic analysis from the results obtained, it can be seen that the system of basic processing and gives the Chinese herbal compound decoction of Sijunzitang retained the chemical composition, in which junior high school drug pieces own ingredients. There is also part of the reaction and the hydrolysis products, such as glucuronic acid and glycyrrhetinic acid. These two components are generated after the glycyrrhizic acid hydrolysis [11], glycyrrhetinic acid has antitussive 
Citation: Zhang L, Zhao H, Zhou G, Niu T, Yang J (2010) Simulation Database System of the Active Ingredients in Compound Decoction of Chinese Medicine. J Bioequiv Availab 2: 131-134. doi:10.4172/jbb.1000045

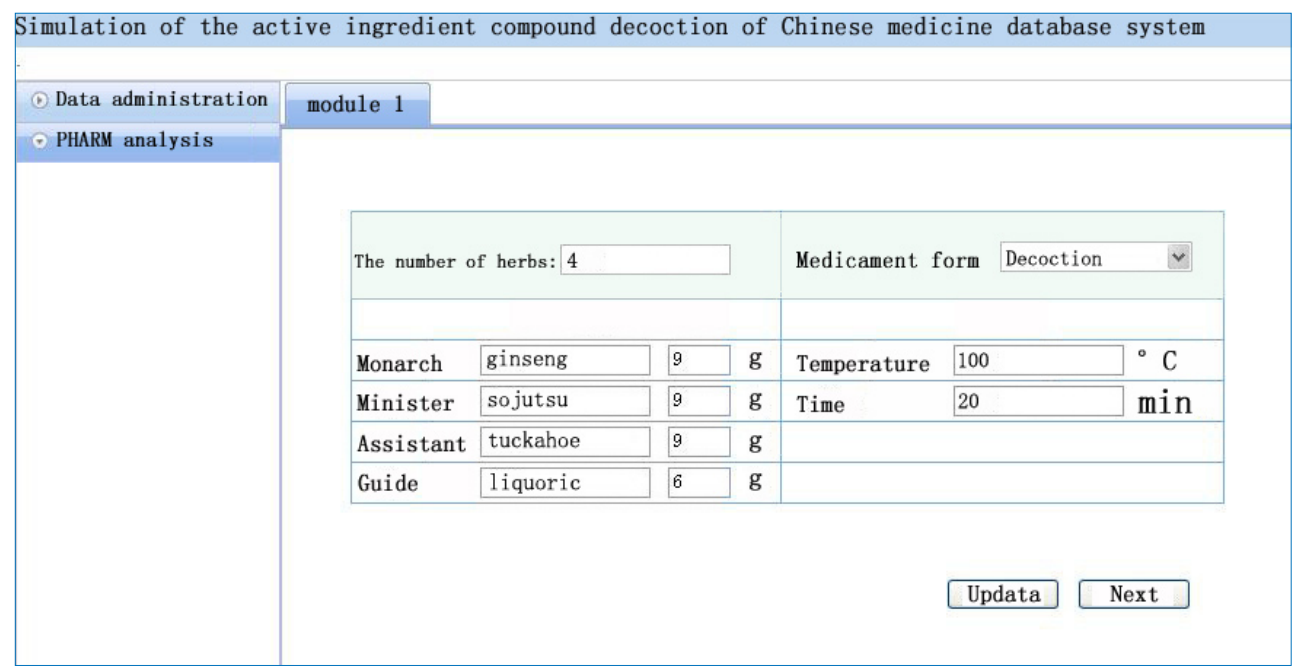

Figure 2: Interface of Chinese medicine compound decoction.

\begin{tabular}{|c|c|c|c|c|c|}
\hline \multicolumn{6}{|c|}{ Simulation of the active ingredient compound decoction of Chinese medicine database system } \\
\hline (1) Data administration & module 1 & & & & \\
\hline \multirow{3}{*}{ - PHARM analysis } & Ginseng & Sojutsu var & Tuckahoe & Liquorice & $\hat{\underline{1}}$ \\
\hline & $\begin{array}{l}\text { Ginsenoside R0 } \\
\text { Ginsenoside Ra1 } \\
\text { Ginsenoside Ra2 } \\
\text { Ginsenoside Rb1 } \\
\text { Ginsenoside Rb2 } \\
\text { Ginsenoside Rb3 } \\
\text { Ginsenoside Rc } \\
\text { Ginsenoside Rd } \\
\text { Ginsenoside Re } \\
\text { Ginsenoside Rf }\end{array}$ & $\begin{array}{l}\text { Atractylone } \\
\text { Atractylol } \\
\text { Aromadendrene } \\
\text { Atractylenolide } \\
\text { I, } 3 \\
\text { Atractylenolide } \\
\text { II, } 4 \\
\text { Atractylenolide } \\
\text { III, } 5 \\
\text { Biatractylolide }\end{array}$ & $\begin{array}{l}\text { Daueosterol } \\
\text { Ergosta- } 7,22- \\
\text { Diene-3 } \beta \text {-ol } \\
\text { Pachymic acid } \\
24 \text { (31) -trien- } \\
21 \text {-oic acid } \\
3 \text { B, 16 a-dihy- } \\
\text { droxylanosta-7, } \\
9\end{array}$ & $\begin{array}{l}\text { Glycyrrhetinic acid } \\
\text { Liquifitigenin } \\
\text { Isoliquiritigenin } \\
\text { Liquiritin } \\
\text { Isoliquiritin } \\
\text { Neoliquiritin } \\
\text { Neoisoliquiritin } \\
\text { glycyrol } \\
\text { Licoricidin } \\
\text { glycyrrhizi acid }\end{array}$ & $\underline{v}$ \\
\hline & & & & Back Next & \\
\hline
\end{tabular}

Figure 3: Interface of active of Chinese medicine compound and their interactions.

and treatment of gastric ulcer and so on. As the program will set the reaction conditions of $100^{\circ} \mathrm{C}$ water-soluble, so the system rounds the non-water-soluble components and volatile oils.

\section{Discussion}

The effective components of Chinese medicine compound decoction simulation database system, through the existing database of information retrieval, analysis, have been able to derive the initial compatibility of Chinese medicine compound in the chemical composition. This may occur in laboratory experiments to provide some reference for the work, but because of the current effective components of the simulation database system are still under construction, so the initial system's functionality is still relatively simple. The database is also not perfect, and further changes will be needed for the data structure, retrieval speed and functionality to improve it further, and to increase the pharmacological pathology database to bring together relevant data on the role of Chinese herbal medicine as a more accurate simulation, so that the system really can research Chinese medicine to provide some help.

\section{Acknowledgements}

This work was jointly supported by the Proficient Talent Project of Lanzhou Institute of Chemical Physics, Chinese Academy of Sciences (Grant No: 070430SRC1) and the Scientific Innovation Project of Northwest Normal University (Grant No: NWNU-KJCXGC-03-57, NWNU-KJCXGC-03-49).

\section{References}

1. Zhao GF (2009) Prunella vulgaris compound and its clinical research. Chin J Modern Applied Pharmacy 26: 1124-1126.

2. Zhang FC, Chen M, Yan YD (2008) Modern decoction method vs. traditional decoction method. Chin. Pharmacy 19: 478-480.

3. Xu FY, Huang XR (2009) Research progress of extraction technique of TCM Chin. J.Information Tradit Chin Med16: 88-89. 
Citation: Zhang L, Zhao H, Zhou G, Niu T, Yang J (2010) Simulation Database System of the Active Ingredients in Compound Decoction of Chinese Medicine. J Bioequiv Availab 2: 131-134. doi:10.4172/jbb.1000045

4. Shi QS, Liu TQ (2009) Progress in separation technologies of the active ingredients from Chinese medicine. Sci Techno Innovation Herald 15:9

5. Liu YX, Wang SL, Guo J, Sun YT (2008) Review on extraction method of traditional Chinese medicine. Chin Pharmaceutical 17: 2-3.

6. Wei G (1998) Standards of decoction. Chin J Information Tradit Chin Med 5: 6-7.

7. Yuan SS, Wang XL, MA SP (2007) Construction and research on TCM special database. Pharmacy Edu 23: 41-42.
8. Hu JJ (2009) System design on TCM characteristics information. Computer Engineering 35: 269-273.

9. Dai XP, Li JB, Chen S (2006) Effect on lymphocytes function in spleendeficiency-syndrome rat by Sijunzi decoction. Chin J Modern Med 16: 12881289.

10. Zhang QR (2007) Pharmacological research of Sijunzi decoction interacted with gastrointestinal system. Chin J Tradi Veterinary Sci 35: 34-37.

11. Sun XY, Huang LJ, Liu X (1994) Chemical composition analysis of Glycyrrhiza. Acta Chin Med Pharmaco 22: 40-42. 